\title{
Labyrinthe
}

25 | 2006 (3)

La Bande dessinée : ce qu'elle dit, ce qu'elle montre

\section{Boilet et l'envers de la Bd}

Nouvelle manga ou dernier avatar du japonisme?

\section{Guillaume Paugam}

\section{(2) OpenEdition}

Journals

Édition électronique

URL : http://journals.openedition.org/labyrinthe/1415

DOI : $10.4000 /$ labyrinthe. 1415

ISSN : 1950-6031

Éditeur

Hermann

\section{Édition imprimée}

Date de publication : 1 décembre 2006

Pagination : 83-96

ISBN : 2-9526131-2-5

\section{Référence électronique}

Guillaume Paugam, « Boilet et l'envers de la Bd », Labyrinthe [En ligne], 25 | 2006 (3), mis en ligne le 28 mars 2010, consulté le 02 mai 2019. URL : http://journals.openedition.org/labyrinthe/1415; DOI : 10.4000/labyrinthe.1415

Ce document a été généré automatiquement le 2 mai 2019.

Propriété intellectuelle 


\title{
Boilet et l'envers de la Bd
}

\author{
Nouvelle manga ou dernier avatar du japonisme?
}

\author{
Guillaume Paugam
}

1 Un exemple parmi d'autres: Les Mondes Manga ${ }^{1}$, à l'instar de tout récent ouvrage un peu panoramique sur le sujet, entre visite au musée du manga de Kawasaki, présentation de séries adolescentes emblématiques et autres descriptions illustrées de cosplay, réserve un chapitre à Frédéric Boilet. Sans que l'on sache très bien si c'est pour son œuvre propre, son installation au Japon et rôle subséquent de passeur entre les deux cultures ou sa tonitruante prise de position contre le manga commercial, peut-être un peu pour tout cela, Boilet est désormais presque une institution. À une telle exposition l'auteur se prête d'ailleurs fort bien, payant volontiers de sa personne pour composer ses albums où réalité et fiction prétendent constamment se confondre ; jouant de son personnage pour imposer un combat dont on ne sait plus très bien s'il est d'avant- ou d'arrière-garde et qui ne transparait plus que dans la distinction grammaticale qui le porte («la» manga d'auteur contre « le » manga industriel). La permutation des genres prêtant à sourire, elle pourra être reprise, mais sous forme de clin d'œil et de manière parfaitement anodine: si Les Mondes Manga l'adopte, c'est en la désamorçant soigneusement par des guillemets et uniquement pour parler de Boilet, quitte à mêler partout ailleurs les torchons Naruto aux serviettes Tezuka. Qu'importe, l'auteur est situé.

2 Cette définition kaléidoscopique rend peut-être d'autant plus nécessaire une approche de son travail déprise des poncifs qui surlignent intempestivement l'entrée «Boilet » au registre des iconoclastes - tentative qui passe par la recherche d'une cohérence graphique, scénaristique, voire biographique de l'œuvre. À ce titre, il y a un véritable intérêt à relire, presque dix ans plus tard, l'album Tôkyô est mon jardin ${ }^{2}$ à la lumière du livre L'Apprenti japonais ${ }^{3}$. Dès l'ouverture, l'épigraphe du second (une dédicace à Dominique Noguez, auteur de la très généreuse préface au premier) y invite, formant très explicitement le trait d'union entre les deux œuvres:

Pour les besoins de la cause, notre auteur poussera l'abnégation [...] jusqu'à tomber amoureux, puis à vivre avec son héroïne, je veux dire avec le glorieux modèle de celle-ci. D'où ces rituels préalables que l'on trouvera dans ses carnets et ses photos s'il consent à les publier un jour... (Tôkyô est mon jardin, 1997). 
À Dominique Noguez, ces quelques notes et croquis forcément modestes, mais qu'il

a été le premier à lire, puis à m'encourager à publier... C'était il y a douze ans ( L'Apprenti japonais, 2006). nu. Par là il s'agit moins, on s'en doute ${ }^{6}$, d'effacer les contributions respectives de Benoit Peeters (co-scénariste) et Jirô Taniguchi (crédité pour les trames) dans la composition de Tôkyô est mon jardin que de donner lieu à une œuvre singulière par sa dé-composition. Au delà, le jeu des dates entre l'observation de terrain ${ }^{7}$ et la parution tardive de ces impressions de voyage permet un parcours à double entrée où œuvre et vie s'entremêlent: c'est en regard de l'album Tôkyô est mon jardin où ils apparaissaient transfigurés par la narration que l'on peut retracer l'itinéraire des fragments de vie consignés par L'Apprenti japonais, mais la particularité de l'auteur Boilet n'est-elle pas mieux cernée encore au vu de sa démarche artistique personnelle qui va des élaborations scénaristiques de Tôkyô est mon jardin au dépouillement narratif de L'Apprenti japonais? Bien étrange va-et-vient entre ces deux œuvres où la première est à la fois passé (composé) et futur (antérieur) de la seconde, la seconde, prolongement et source de la première... De sorte que L'Apprenti japonais paraît au final plus qu'une simple exhibition du matériau de Tôkyô est mon jardin : une véritable expérience d'écriture autant que de vie qui, entre roman expérimental et récit de voyage, trace au fil du pinceau son chemin plaisant et exotique; presque un aboutissement. L'Apprenti japonais se présente ainsi ouvert à trois types de lecture : en tant qu'ouvrage isolé, puisque ce recueil offre une sorte de rapport d'activité pour les treize dernières années japonaises de son auteur (des premières observations de voyageur aux dessins de presse parus dans un grand quotidien); en tant que document, car sa composition permet de mieux appréhender la méthode Boilet (ainsi, des photographies originales sont ici reproduites qui donneront lieu à des scènes redessinées pour les albums, divers faits rapportés qui seront rescénarisés, etc.); en tant que clef de compréhension de l'œuvre, si l'on admet que la publication aujourd'hui de ces notes anciennes, indépendamment de leur caractère informatif (biographique et documentaire, donc) fait sens. C'est cet aspect qui nous intéresse ici.

5 Mais peut-être faut-il d'abord justifier l'intégration paradoxale de cette œuvre au sein d'un opus de Boilet. Aussi éloigné qu'il soit d'un album de bande dessinée, le recueil formé par L'Apprenti japonais peut en effet être inscrit dans la continuité des productions antérieures de Boilet dont il parachève les procédés. L'évolution du travail de l'auteur apparaît ainsi rétrospectivement à travers la dimension diaristique affectée par L'Épinard de Yukiko ${ }^{8}$, l'appauvrissement progressif du narratif au regard du dessin, le rapport toujours plus ténu à l'autobiographie. 
6 Dans L'Épinard de Yukiko, les reproductions de pages d'agenda qui scandent l'histoire, l'ancrant dans un temps à la fois réaliste et oppressant (entre le 8 avril et le 28 mai), entretiennent avec le récit un rapport ambivalent. Distrayantes ou explicatives à l'occasion, elles peuvent aussi se montrer tout simplement tautologiques vis-à-vis du développement narratif, le réduisant parfois au rôle d'illustration du journal. Ainsi la fictive (? $)^{9}$ - page 14 en regard de la page 15 : «Vernissage de l'expo Nouvelle Vague au Bunkamura [...], ai rencontréYukiko Hashimoto. Ellem'a souri.» S'ensuit une planche dessinée de l'avenante jeune femme en question et de son sourire. Dont acte. L'Apprenti japonais ne fera pas autrement - à ce détail près que les photographies de Boilet qui soustendaient un travail pictural orienté vers toujours plus de réalisme remplaceront alors ces dessins à valeur purement illustrative.

7 Par ailleurs, avec L'Épinard de Yukiko, qualifié dans les premières pages de Mariko Parade de «quasi-documentaire» (p.11), David Martin, personnage principal de Boilet-Peeters depuis Love hôtel ${ }^{10}$ jusqu'à

Demi-tour ${ }^{11}$, disparait. Il fait placeàun « mangaka »(L'Épinard de Yukiko, p.136) dont les traits sont empruntésàBoilet lui-même. Cetteforme de continuité est bien sûr problématique: de deux albums aussi différents que Tôkyô est mon jardin et L'Épinard de Yukiko, où les personnages, l'histoire, le dessin, la technique narrative ne sont pas les mêmes, comment peut-on dire que l'un est la suite de l'autre ? Paradoxalement, c'est L'Apprenti japonais qui permet de réinventer une continuité, où l'on voit que les notes de Boilet, qui jusqu'alors étaient exploitées pour le compte de l'un, seront désormais versées au compte de l'autre. C'est ainsi qu'en sous-main le double effacement du récit (au profit du « réel », d'un réel non décrit mais présenté au gré de son matériau composite) prépare la mise à l'écart du narratif accomplie par L'Apprenti japonais au profit du seul journal (pages d'agenda, extraits de fax et de correspondance), désormais assumé à la première personne et situé dans la réalité, spatiale et temporelle, de Boilet.

Plus qu'une simple continuation, L'Apprenti japonais semble ainsi marquer, dans la production de son auteur, le passage d'une auto-présentation («spéculaire » car mettant en scène un auteur de bande dessinée) au pur et simple journal. La confusion entre vie et œuvre de l'auteur se fait alors plus insistante et revendiquée que jamais, mais ses termes étant posés plus honnêtement, c'est la question de l'évolution de l'auteur Boilet qui affleure de cet acte inattendu qui répond à distance au geste accompli précédemment par L'Épinard de Yukiko.

Le passage de Tôkyô est mon jardin à L'Épinard de Yukiko était en effet celui d'un auteur écrit (le personnage de David Martin, ex-employé de ministère, représentant en alcool et manœuvre au marché aux poissons de Tsukiji, nourrissait des velléités romanesques ${ }^{12}$ ) à un auteur écrivant : un Boilet transposé, maître d'œuvre de sa propre histoire. D'où cette étrange temporalité du récit, ce présent alangui par les redondances d'un dessin qui mêlait trait abouti (L'Épinard de Yukiko, p.18-19), crayonné sur agenda (p.68) et « encré » sur ordinateur (p.69 et 71), alangui par la litanie des dialogues recommencés qui scandaient le récit (le dialogue inaugural et son homophonie éponyme o heso-nombril/ hôrensô-épinard [p. 8-12, p. 54-57, p. 127-131]; l'avertissement mécanique au sortir du tapis roulant [p. 34], revenant de plus en plus insistant, prémonitoire même [p.74-75], menaçant [p.98-99]). La mise en abyme elle-même en venait à former une sorte de mélopée visuelle au mystère insaisissable - les huit dernières planches de l'album marquaient tout à la fois la fin du récit et le début d'une autre histoire, expliquant le changement de modèle au mitan du livre (p.61) au détour d'une scène "réelle " 
(p.135-139) fictionnalisée peu avant (p.101-103) - où la confusion des modèles, qui rendait la fiction invraisemblable ${ }^{13}$ en rendait plus crédible le vécu. En désynchronisant à dessein « image » et «son ", l'auteur recréait ainsi la perception déformée de son narrateur que travaillait le souvenir douloureux d'un amour voué à l'échec au moment de mettre en scène sa propre histoire et sa mélancolie à l'écrire.

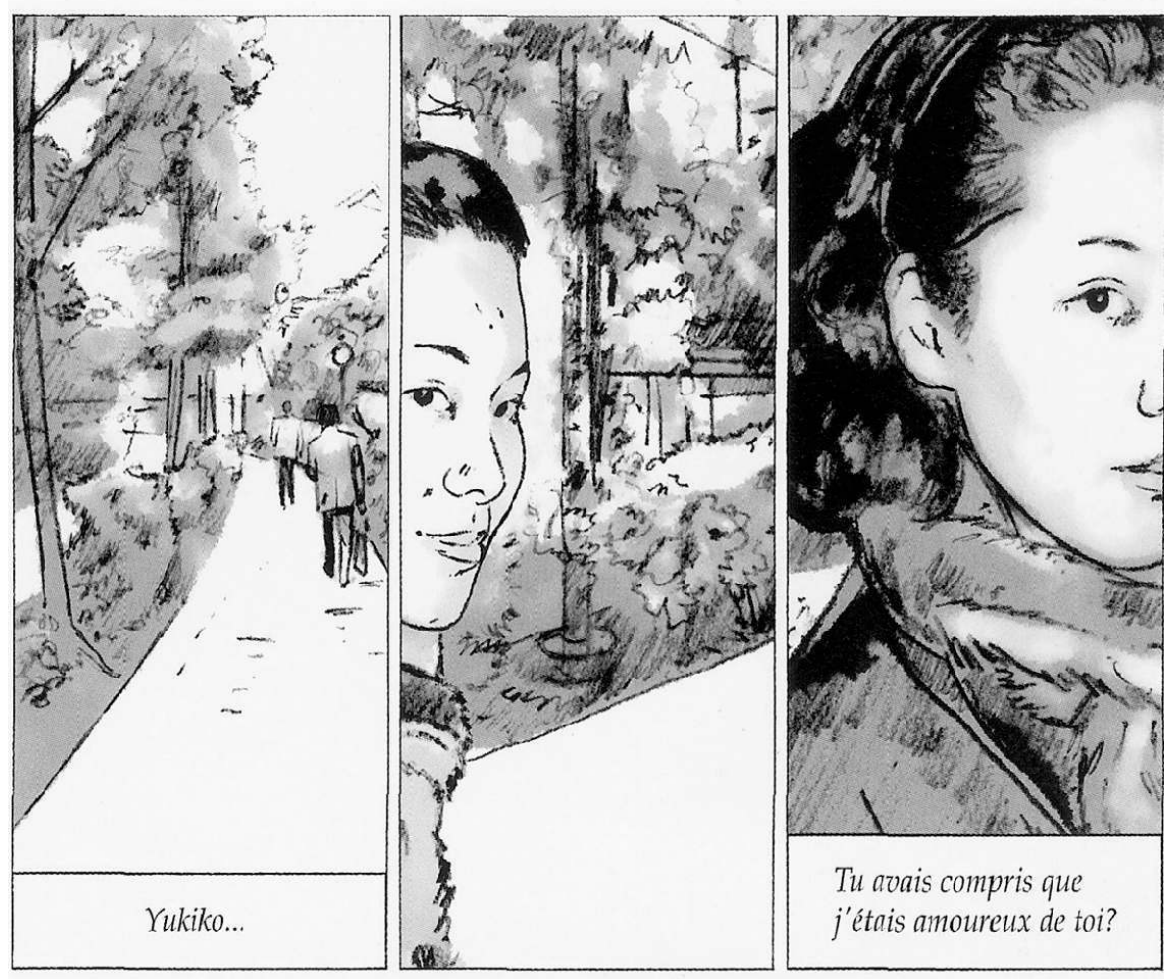

L'Épinard de Yukiko, Frédéric Boilet@ Ego comme X.

11 Avec L'Apprenti japonais, Boilet décide cette fois d'exposer l'avant-scène de l'écriture. Il se tourne ainsi vers le passé et, avec la publication de ce matériau qu'il avait laissé pardevers lui, s'accule dans une régression sans issue. Délibérément, il semble organiser l'exhibition ultime après laquelle il ne lui restera plus rien à explorer. Aussi, bien que L'Apprenti japonais soit empreint d'alerte légèreté ${ }^{14}$, le fait de s'en dessaisir peut conduire le lecteur à regarder avec distance les sentiments exprimés, comme autant d'illusions perdues. Peut-être faut-il en conclure que Boilet est parvenu à une forme d'achèvement dans sa quête. En tout état de cause, L'Apprenti japonais marque indiscutablement une étape dans son parcours.

Du rôle de «gugusse » (Love hôtel, p. 65) de service à celui de guide idoine pour Tôkyô est mon jardin, David Martin avait pris du galon jusqu'à affecter une parfaite connaissance du Japon, de ses mœurs nocturnes, de sa géographie urbaine, de son impétuosité météorologique et tellurique : "Vous êtes ici comme un poisson dans l'eau ", lui lançait son employeur alors en visite (Tôkyô est mon jardin, p. 93) précipitant bientôt son retour pour cause de typhons et autres tremblements de terre. L'Apprenti japonais en révélant la facticitédu flegme affiché(Boilet,moins flegmatique que son personnage: «j'ai passé la nuit l'œil collé à mon téléviseur [...] toute cette agitation médiatique n'a pas perturbé une seconde le sommeil de Kaoru ", p. 59-61) lève un peu du voile enveloppant l'identification imparfaite de Frédéric Boilet au personnage de David Martin et pose en amont la question de l'identité de son auteur - problématique que le titre même d'Apprenti japonais désigne : 
Quel que soit le temps passé ici, je resterai toujours un apprenti japonais, quelque part entre le déjà plus vraiment français et le jamais japonais... Me fondre dans la société japonaise, pour y devenir plus japonais qu'un Japonais? Je n'en ai jamais eu l'envie, tu le sais bien, et en serais de toutes façons bien incapable ${ }^{15}$ !

Hors les faux-fuyants des constructions en abyme, par la mise à l'écart de tout procédé BD, L'Apprenti japonais désigne sans plus d'ambiguïté son auteur et la nature des préliminaires qu'il met en œuvre. C'est ce fonds d'impressions et d'idées préconçues, l'arrière-cuisine de l'œuvre, la pré-bande mobilisée par l'auteur qu'il faut à présent interroger pour comprendre la solennité mise à le dévoiler - ce en quoi L'Apprenti japonais sembleàla fois le point d'orgue de son travail et un adieuàl'écriture où ce qui fait la cohérence de l'œuvre est aussi ce à quoi l'auteur a renoncé.

Or, de la fièvre exploratoire préalable à Tôkyô est mon jardin, L'Épinard de Yukiko et même Mariko Parade cette avant-scène de la bandedessinée que L'Apprenti japonais révèle, ce qui reste surtout c'est la candeur d'un Boilet envers un Japon réduit singulièrement à ses habitantes ${ }^{16}$, géographiquement à sa métropole ${ }^{17}$. Au cœur de sa démarche d'auteur, plus que le Japon, c'est le désir de Japon qui semble alors motiver le travail d'un Boilet tout à la fois au plus proche et irréductiblement éloigné de l'objet de ses attentions, perpétuellement en quête d'un émerveillement inédit dont les prolégomènes rameutent pourtant sans discrétion les mêmes inlassables clichés :

Les Japonaises sont formidables. Elles [...] ne vous prennent pas de haut et ne débarquent pas avec leurs problèmes comme les Françaises, sont là sans bouger avec l'air de vous attendre, sont toujours d'accord pour faire l'amour, s'extasient au moindre mot gentil ou quand vous leur tenez la porte (L'Apprenti japonais, p.106-107).

Élevées comme des enfants, elles en ont le caractère et tout le naturel, puis elles sont avenantes, toujours polies, toujours gaies, souriantes, pleines de gentillesse et de l'humeur la plus facile ${ }^{18}$.

Ainsi, la rencontre d'un Français et d'une Japonaise, c'est la rencontre de deux personnes habituées à faire des efforts, et toute surprises de voir l'autre venir à soi. Entre ces deux-là, c'est un peu comme des vacances (L'Apprenti japonais, p.121).

Égoïstes et vaniteux que nous sommes, nous voilà trop ravis de devenir les esclaves de ces savoureuses esclaves. Avec elles, la vie est simple et facile, sans complications, sans subtilités, sans cas psychologiques, sans controverses, sans problèmes à résoudre, sans luttes à soutenir ${ }^{19}$.

C'est avec une fascination trouble que l'on reconnait, sous les mots anodins et personnels de Boilet, l'ordre d'un discours depuis longtemps connu. Et si l'on peut qualifier l'attirance irréfléchie de Boilet pour le pays de «la manga » de japonisme, c'est sur le modèle de l'antiaméricanisme tel que conçu par Philippe Roger où les propos individuels, réactivant leur éternel assortiment de clichés, provoquant sans fin la rémanence des mêmes images, composent à travers les âges et en dépit des particularités de ses instigateurs un ensemble relativement homogène de topoï. Ainsi la philia de Boilet ne lui est pas si exclusive qu'elle ne puissese voir inscrite et mise au compte de la longue tradition du japonisme où - tel le «bloc sémiotique historiquement stratifiée 20 » du discours «anti» - les figures convenues du discours amoureux oblitèrent immanquablement la singularité de ceux qui le tiennent.

Beaucoup de ces notes prises au vol par Boilet évoquent ainsi les diverses strates du japonisme français, de ces livres curieux de grands voyageurs sur les traces de Loti recueillis par Patrick Beillevaire au tourisme intellectuel ou «second japonisme» des 
Kojève, Sartre, Barthes et Lacan au mitan du xx siècle, s'emmaillant en un texte unique, anonyme et atemporel, partageant un même étonnement émerveillé :

Les Japonais dorment beaucoup, n'importe quand, et surtout n'importe où: dans le métro, dans la rue, sur un banc, par terre, sur leur vélomoteur...

la descente dans le sommeil (que l'on peut observer sur tant de visages, dans les trains et les métros du soir $)^{21} \ldots$

... ou s'emportant contre un ennemi commun :

Un paradoxe surprenant est que cette censure [de la commission Eirin créée en juin 1949 selon le modèle du code Hays et sur proposition du Quartier général américain], bien qu'issue du puritanisme catholique [sic] américain, agisse au pays des shunga (estampes japonaises érotiques) et de Nagisa Ôshima. [...] Et avec quelle indécence ! En marquant l'œuvre des sceaux de l'infamie, Eirin rend «suspecte» la moindre scène de nu, « obscène ", la moindre scène d'amour. Au final, c'est elle qui engendre un film " pornographique "!

Les Japonais nous ont tout emprunté : ils ont commencé par les canons, ils ont continué par la redingote et le chapeau haut de forme, et sont en train de finir par notre pudibonderie polissonne et jésuitique. [...] Plus voisins de nous de l'âge d'or qui, hélas ! pour le Japon touche à sa fin -, les Japonais sont restés fidèles aux idées de leurs ancêtres: le nu n'est pas obscène. Mais notre civilisation, pénétrant à grands pas, les oblige, peu à peu, à modifier leurs costumes et leurs idées morales. [Les société évangéliques anglo-américaines] se démènent maintenant pour faire supprimer les bains mixtes ${ }^{22}$.

18 Boilet, d'esprit fantasque et connaissant ses classiques ${ }^{23}$, joue de manière implicite avec la référence barthésienne :

[Boilet :] Kuuzenzetsugo: sans précédent et pas renouvelable! Uttori suru: rester en extase..

[Barthes:] Ouvrez un guide de voyage: vous y trouverez d'ordinaire un petit lexique, mais ce lexique portera bizarrement sur des choses ennuyeuses et inutiles [...]. Cependant, qu'est-ce que voyager? Rencontrer. Le seul lexique important est celui du rendez-vous.

rendez vous yakusoku

tous les deux futaritomo

où? doko ni?

quand? itsu ${ }^{24}$ ?

19 Boilet et Barthes - Claude Coste l'a suffisamment montré ${ }^{25}$ - ont en effet en partage la motivation sexuelle de leur circuit touristique et ont - comme le confirme Yoshiko Ishikawa dans le même Magazine littéraire -plus fondamentalement en commun le désir d'authenticité(voire « autochtonicité ») pour leurs rencontres :

Parlant de Stendhal en Italie, Barthes se demande ce qu'est l'amour d'un pays étranger, et laisse paraître sa propre passion pour le Japon [...] lorsqu'il montre de la sympathie à Stendhal qui raconte le désagrément de rencontrer un compatriote dans le pays adoré, je me figure le sentiment de Barthes quand, à Tôkyô, il logeait à l'Institut franco-japonais... les gaïin, ce sont les étrangers, et je fais justement mon possible pour ne pas les rencontrer, évitant les rendez-vous à l'Institut francojaponais ou me faisant porter pâle les dites soirées. Satoko ne pouvait pas le deviner ${ }^{26}$.

Les voyageurs du début du siècle ne pensaient guère autrement qui recommandaient :

Il ne faut pas voir le Japon là où il est modernisé, dans les ports ouverts aux navires européens, comme Kobé, Yokohama ou Nagazaki. Il faut aller dans les petits villages de pêcheurs cachés au fond des criques de la côte japonaise, ou dans les petites villes situées dans l'intérieur des terres, que des routes bicyclettables vous 
permettent d'atteindre facilement. Là, l'indigène a gardé son caractère original et ne connaît pas encore le soulier verni et le chapeau de soie ${ }^{27}$.

21 Les poncifs n'évoluent d'ailleurs que rarement au rythme des mutations réelles de leur objet fantasmé :

Dans tous les cas, ce soi-disant progrès enlèvera au Japon de plus en plus son originalité et le rendra de moins en moins intéressant au voyageur.

Le Japon entre dans la mue occidentale: il perd ses signes, comme on perd ses cheveux, ses dents, sa peau; il passe de la signification (vide) à la communication (de masse).

Chose remarquable, l'atmosphère de ces rencontres hebdomadaires, toute de retenue et de concentration, contraste fortement avec celle de ma vie tokyoïte, pour le moins électrique et débridée. Mais c'est ce même contraste auquel on est confronté à longueur de journées dans un Tôkyô où coexistent, en curieuse intelligence, tradition et modernité ${ }^{28}$.

De tels exemples de conformation de Boilet au discours du japonisme français sont nombreux. Non que l'Orient, où les Européens découvrirent jadis le salpêtre, ne soit pire ou meilleur endroit qu'ailleurs pour inventer la poudre. Mais dans ce pays qu'il ne fait alors que visiter, déjouant les clichés du tourisme de masse (et sacrifiant par-là même au mythe du caractère inédit de leur dénonciation ${ }^{29}$ ), Boilet veut s'oublier... et se montre en cela bien français, tant le désir d'assimilation est caractéristique de ses compatriotes ${ }^{30}$. Boilet est-il pour autant dupe de la teneur identitaire de sa vision du Japon ? Elle affleure à travers le résumé que fait David Martin/Frédéric Boilet à Kimié/Kaoru de l'intrigue de son roman dans Tôkyô est mon jardin :

... Au début, il croit que c'est le Japon qu'il veut comprendre, et peu à peu, il

découvre que le Japon n'est qu'une façon pour lui de se retrouver... (p. 52).

Pour ce qui est de L'Apprenti japonais, l'omniprésente problématique, nonobstant la dénégation peu convaincue affichée par Boilet, se résout presque à l'insu de son auteur, au travers des remarques anodines, des observations in situ, des étonnements ad hoc d'un Boilet estampillé gaulois en chacun de ces prompts jugements et finalement mieux caractérisé encore par ce qu'il ne dit pas.

La révolution avortée contre «le Café froid en boîte » (L’Apprenti japonais, p. 114) rappelle ce mot de Barthes, encore, observant quel'identité, le corps, passe par un certain nombre de choix plus ou moins conscients, par le goût :

Français par les fruits (comme d'autres le furent «par les femmes») : goût des poires, des cerises, des framboises; déjà moindre pour les oranges; et tout à fait nul pour les fruits exotiques, mangues, goyaves, lichees ${ }^{31}$.

Boilet avait-il dès l'abord le goût des fruits exotiques? et des «femmes »? Sa fascination élective est connue, déclarée, affichée, mais moins par «les ${ }^{32}$ » Japonaises que par la Japonaise, singulier hypostasié au nom d'une essence de la race et dénomination plus générale encore à l'ombre de laquelle elles finissent par se ressembler toutes: dans L'Épinard de Yukiko, on l'a dit, Mariko succède sans heurt narratif-si ce n'est le changement de coiffure (p.62), mais au nom d'une ressemblance suspecte (« vous avez le même tee-shirt à rayures!» p.139) - à Yukiko, l'une et l'autre, en leur fonction de modèle, parfaitement interchangeables. 


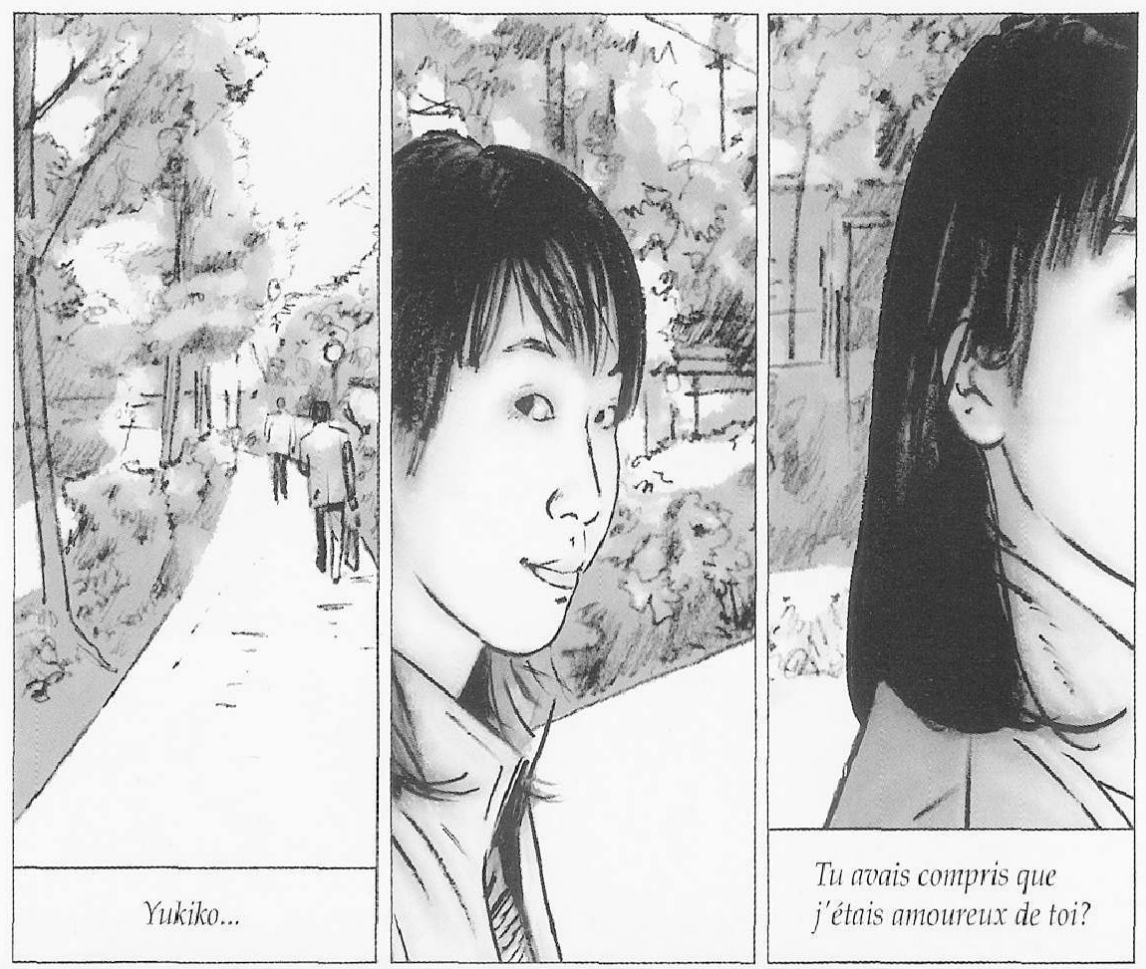

L'Épinard de Yukiko, Frédéric Boilet@ Ego commeX.

Sur cet article des femmes, c'est-à-dire du Japon, David Boilet et Frédéric Martin tendent à se confondre, et leurs aventures - Love parade, Tôkyô hôtel, L'Épinard de Mariko, Yukiko est mon jardin - àapparaitre comme la scène équivoque d'une continuité vie/œuvre à peine déformée. Ainsi dans Love hôtel, David Martin lançait à son interlocutrice francophone : "Vous voyez, l'idéal, c'est une femme qui ait toute la séduction d'une Japonaise, mais à qui je puisse parler comme à une Française» (p. 97); ce que par la bouche de Boilet, Aurélia Aurita traduit dans sa si particulière poétique encensée par la presse mainstream :

Eh bien toi, tu as peut-être un corps d'Asiatique...

... mais tu es bel et bien française !

On peut dire que tu as les avantages des deux !

Toi, tu sens toujours bon, comme une Japonaise...

... mais tu te laisses enculer comme une Française ${ }^{33}$.

De cette nouvelle histoire d'amour, faut-il comprendre que la synthèse-Aurita représente l'ultime raffinement du Japon conçu par Boilet? Est-ce pour cela que Boilet, ayant suffisamment fait le deuil de ses illusions pour publier L'Apprenti japonais, cherche maintenant, en organisant le voyage d'auteurs amis, à retrouver par procuration son naïf émerveillement des premiers jours ? C'est peut-être là le sens du tautologique Japon ${ }^{34} \mathrm{et}$ de la propre contribution de Boiletàce recueil : un savant travelling qui commence aux abords de la ville, chemine en extérieur etfinit dans sa chambre, sorte de close-up de plus en plus intime qui finit sur un plan d'Aurita à ses pieds. 


\section{NOTES}

1. H.-M. Delpierre, J. Schmidt, Tours, E.p.A, 2005.

2. F. Boilet \& B. Peeters, Paris, Castermann, 1997.

3. F. Boilet, Bruxelles, Les Impressions nouvelles, 2006.

4. Ces carnets qui forment le premier tiers du livre sont suivis par trois sous-ensembles «Prisonnier des Japonaises »; "L'Encyclopédie illustrée de la jeunesse » et «Le Monde vu du Japon »- recueillant des textes et dessins composés entre 1998 et 2005.

5. F. Boilet et K. Takahama, Mariko parade, Paris, Casterman « Écritures », 2003.

6. La préface de Noguez lui faisait déjà la part suffisamment belle qui comparait le travail de Boilet, pour la structure de ses planches, au Winsor McCay de Little Nemo; pour le cadrage, à l'art du montage de Dziga Vertov, Eisenstein et des frères Coen ; pour son réalisme, si ce n'est pas antithétique, à Stendhal, Flaubert, voire, après réflexion, à Flaubert mais en mieux («plus fringants ou plus ménagers d'autrui »), etc.

7. «Mais pour préciser les circonstances de mon séjour à Tôkyô, et avec naïveté ou prétention, j'aurais pu inscrire "ethnologue"! Un ethnologue mercenaire, sans formation, qui marcherait au flair, à l'instinct, "au radar", et dont la thèse prendrait la forme d'une narration... », L'Apprenti japonais, op. cit., p. 33.

8. F. Boilet, Angoulême, Ego comme X, 2001.

9. Sur cette page de L'Épinard de Yukiko figure: «Un type qui est dans l'art, mais pas dans les manières!» (p. 14 - datée du samedi 8 avril [2000]), soit la même notation que pour la première page d'agenda reproduite dans L'Apprenti japonais (p. 27 - datée du 22 mai 1993).

10. Paris, Casterman « Les romans (à suivre) », 1993.

11. Dupuis « Aire libre », 1997.

12. Tôkyô est mon jardin, p. 37, 52-53 et 129 .

13. Le narrateur désigne les imperfections de Yukiko, sa « cicatrice de varicelle, [sa] ride sur la paupière... » (p. 103), alors qu'il dessine précisément Mariko, son modèle de substitution!

14. "[Tôkyô est mon jardin] s'inspire de mon séjour d'une année à Tôkyô en 1993, qui fut particulièrement heureux... J'y ai rencontré le futur modèle du personnage de Kimié, elle ne m'a pas quitté depuis...", L'Apprenti japonais, p. 5. Texte extrait d'un entretien par courrier électronique avec F. Boudet repris en forme de liminaire pour la première partie - «notes, lettres, articles, croquis et photos »- de L'Apprenti japonais.

15. «Extrait de courriel à Benoît Peeters, 15 mars 2006 », dans L'Apprenti japonais, p. 239.

16. «Les Japonaises sont formidables, je vous l'ai déjà dit, et si ça ne tenait qu'à moi, je ne parlerais que d'elles dans ces chroniques", ibid., p. 121. Les chroniques en question sont recueillies sous le titre explicite de « Prisonnier des Japonaises »".

17. «Je passe mes journées à sillonner Tôkyô - car c'est finalement Tôkyô l'objet de mes recherches, plus que le Japon ", L'Apprenti japonais, p. 34.

18. A. de Gabriac, Course humoristique autour du monde. Inde, Chine, Japon, 1872 repris dans $\mathrm{P}$. Beillevaire (Éd.), Le Voyage au Japon. Anthologie de textes français, 1858-1908, Paris, Robert Laffont «Bouquins", 2001, p. 661 (précieuse compilation à laquelle ce court relevé est évidemment hautement redevable).

19. L. Naudeau, Le Japon moderne. Son évolution, 1909 repris dans Le Voyage au Japon, p. 692.

20. «Seule la complexité de ses stratégies entrecroisées [...] lui confère l'allure trompeuse d'un bouquet de lubies individuelles. Les lubies sont là, n'en doutons pas, elles donnent chair aux mots et nerf aux phrases. Mais le discours antiaméricain les met à la masse », dans Ph. Roger, L'Ennemi 
américain. Généalogie de l'antiaméricanisme français, Paris, Seuil,« La couleur des idées », 2002, p. 18-19.

21. F. Boilet, L'Apprenti japonais, p. 53 et R. Barthes, L'Empire des signes dans Euvres complètes, t. III, 1968-1971, (éd. É. Marty), Paris, Seuil, 2002 [1970].

22. F. Boilet, L'Apprenti japonais, p. 84-86 et J.-J. Matignon, L'Orient lointain. Chine, Corée, Mongolie, Japon. Impressions et souvenirs de séjour et de tourisme, 1903 repris dans Le Voyage au Japon, p. 653 et 657-658.

23. Dans une note ultérieure, Boilet s'autorise un clin d'œil plus explicite à son illustre prédécesseur : « Dans L'Empire des signes, Roland Barthes avait décortiqué le Japon des années 60 ; en l'an 2000, il ne raterait pas la débauche de doigts levés sur pellicules!», extrait d'une des chroniques reprises dans «L'Encyclopédie illustrée de la jeunesse » qui forme l'avant-dernière partie de L'Apprenti japonais, p. 182.

24. F. Boilet, L'Apprenti japonais, p. 26 et Roland Barthes, L'Empire des signes, p. 360-361. On pourrait discuter l'orientation de cette traduction, ce n'est guère le lieu ici.

25. "Combien de lecteurs de L'Empire des signes ont-ils compris que le petit plan donné en illustration retraçait le circuit des bars homosexuels de Tôkyô ? ", Claude Coste, "Le secret de l'œuvre », dans Magazine littéraire, $\mathrm{n}^{\circ}$ 314, Paris, octobre 1993, p. 30.

26. Y. Ishikawa, «La Passion du Japon », dans Magazine littéraire, op. cit., p. 70-71 et F. Boilet, L'Apprenti japonais, p. 69.

27. J.-J. Matignon, Le Voyage au Japon, p. 654.

28. Ibid., p. 657 ; R. Barthes, L'Empire des signes, p. 426 et F. Boilet, L'Apprenti japonais, p. 54.

29. «Je ne me suis pas offert les services d'une geisha et n'ai croisé aucun samouraï, je n'ai pas assisté à la parade du mariage princier et l'extrême droite nippone a négligé de m'insulter, je n'ai pas visité le temple d'or de Kyôto, je ne me suis pas plus approché des cerfs de Nara, je n'ai passé aucune nuit dans un hôtel capsule ni aperçu un seul pousseur dans le métro de Tôkyô... Mais il y a pire, l'incompréhensible, l'inexcusable négligence, j'ose à peine l'avouer : je quitte le Japon sans avoir vu le Mont Fuji!», F. Boilet, L'Apprenti japonais, p. 99. Barthes écrivait pour sa part: «Souvent, ce qu'il [Barthes lui-même] lui faut déjouer, ce n'est pas la banalité de l'opinion courante, c'est la sienne propre ; le discours qui lui vient d'abord est banal, et ce n'est qu'en luttant contre cette banalité originelle que peu à peu il écrit. [...] (la banalité, c'est le discours sans corps). En somme, ce qu'il écrit procéderait d'une banalité corrigée », dans R. Barthes, Roland Barthes par lui-même, Paris, Seuil «Écrivains de toujours », 1975, p. 141.

30. Dans son pays d'accueil, le migrant se conforme " naturellement » au modèle d'immigration qu'il importe de facto de son pays d'origine. D'où des malentendus qui peuvent surgir lorsque les deux modèles diffèrent trop pour ne pas entrer en tension. Ainsi, l'exigence française d'assimilation (ou du moins sa mise en discours, en France, au sujet des immigrants) imprègne le Français émigrant indépendamment de la conception de l'intégration promue par son pays d'accueil. Je me réfère ici aux travaux de Sophie Fesdjian : «Chez le Français migrant, appliqué et bon élève, se développe donc la ferme intention de s'intégrer pour faire plaisir à ses hôtes. Il ignore cependant que tous les pays n'ont pas la même idéologie et que ses efforts vont bien souvent se heurter au modèle de son pays d'accueil. Aux Pays-Bas, il va se voir reprocher, entre autres, d'apprendre le néerlandais, en Amérique du Nord personne ne tient à partager sa souffrance car un migrant est un citoyen heureux!... ». Voir «Des Bretons à Montréal: Anthropologie contemporaine d'une migration transatlantique ", mémoire de DEA d'anthropologie sociale, Paris, EHESS, 1997.

31. Roland Barthes, p. 100.

32. "Les Japonaises sont formidables. Elles vous regardent droit dans les yeux en souriant... ", L'Apprenti japonais, p. 106 ; Une chose surprenante, par exemple, et qui m'a frappédès mon premier jour au Japon, est cette façon qu'elles ont, quand on les croise dans la rue, de vous sourire et de vous regarder droit dans les yeux... "; ibid., p. 121. 
33. Fraise et Chocolat, Paris-Bruxelles, Les Impressions nouvelles, 2006, p. 133-134.

34. F. Boilet et ali, Japon. Le Japon vu par 17 auteurs, Paris, Casterman « Écritures », 2006.

\section{AUTEUR}

\section{GUILLAUME PAUGAM}

Labylefil[at]gmail.com 